SLAC-PUB-1333

$(\mathrm{E} / \mathrm{A})$

October 1973

\title{
RADIATION MEASUREMENTS IN SECONDARY
}

\section{ELECTRON BEAM AND COMPARISON WITH CALCULATIONS*}

Kenneth R. Kase $\dagger$, Walter R. Nelson and Lewis Keller

Stanford Linear Accelerator Center

Stanford University, Stanford, California 94305

\begin{abstract}
A high energy particle beamline is described which is capable of transporting a high intensity electron beam to potentially occupied areas. Ionization chamber measurements of electron beam intensity are described together with the ion chamber calibration and necessary corrections. The results of the measurements are compared with a Monte Carlo calculation of the electron intensity.
\end{abstract}

(Submitted to Health Physics.)

*Work supported by the U. S. Atomic Energy Commission.

$\nmid$ Present address: Stanford University Medical Center, Radiology Department, Stanford, California 94305. 


\section{Introduction}

The Stanford Linear Accelerator produces an electron beam at energies up to $22 \mathrm{GeV}$. Maximum primary beam power attained to date is in excess of $800 \mathrm{~kW}$. For high energy particle physics experiments performed at SLAC, primary electron beams are generally directed onto a target to produce a high energy secondary particle beam. Primary electron beams are contained in well-shielded enclosures and absorbed in beam dumps or targets designed to operate at the high powers available. The secondary particle beams are transported from the target to the experimental areas which include spark chambers, bubble chambers and particle counters of various sorts. Many and varied safety devices are used to prevent the primary electron beam from entering a secondary beam channel.

In the experiment to be discussed in this paper the secondary particles are muons. The muons are generated by high energy photons in pair production interactions and are strongly peaked in the forward direction. To get maximum muon yields it is necessary for the secondary particle beam to be taken from the target at an angle of $0^{\circ}$ relative to the incident electron beam. This beam geometry establishes a situation in which it is quite likely for the primary electron beam to be directed into the secondary beam channel. Calculations and measurements made to establish the radiation hazards of this beam geometry and the applicability of these results to subsequent experimental beams are discussed in this paper.

\section{Beam Description}

A. Geometry

The primary beam path in which the muon beam is generated is set up so that two beams for two separate experiments can run simultaneously (in a 
pulse-sharing mode of operation). Two experiments using different targets, different particles and different secondary beam paths, are shown in the vertical ray trace (Fig。1). Primary electrons which produce the muon beam are absorbed in the muon production target while primary electrons which produce a pion beam are absorbed in a beam dump which also contains the beryllium filter for the muon beam. The Be filter serves as an absorber for all the particles transported along with muons to that point. It also physically blocks the muon beam channel and prevents the transport of a mis-steered primary electron beam through the muon beam channel.

The muon beam is transported through the shielded personnel exclusion area called End Station B by a series of magnets as shown in the vertical ray trace (Fig. 2). As it leaves End Station B it is about 15 feet above the ground. It is then bent downward to the second focus. An aluminum beam pipe encloses the beam between End Station B and the 40 inch bubble chamber except for a six foot region at the second focus. The second focus is enclosed in a wooden building but personnel access to the beam is required from time-to-time. It is this point of access to the beam which is of primary concern from the standpoint of radiation protection. From the second focus the beam is transported to the bubble chamber. After passing through the bubble chamber the beam goes into a large stack of steel and lead.

\section{B. Normal Operation}

Under normal operating conditions the radiation hazard in this muon beam is minor since the dose rate is of the order of $10 \mathrm{mrad} / \mathrm{hr}$ directly in the beam. Electronic beam sensing devices are used to keep the primary electron beam directed to the proper point on the beam dump. Thus under normal circumstances an electron beam would not be directed onto the beryllium filter in the secondary muon beam channel. 


\section{Potential Non-normal Operation}

Under certain failure modes it is possible to steer the primary electron beam onto the Be filter and align it with the muon beam channel. The $\mathrm{Be}$ is thick enough to absorb the primary electron beam, but the electromagnetic cascade shower established in the Be would generate many electrons which could be transported through the muon beam channel. The muon transport system will accept only a narrow range of momenta about the momentum at which the system is set. Thus only a small fraction of the electrons generated in the Be would be transported to the potentially occupied areas such as at the second focus. However, the primary electron beam current can be quite large (about $10^{14}$ electron/second) and the number of electrons transported can very well be great enough to cause a radiation hazard.

We first estimated the magnitude of the hazard by a simple analytical calculation. Since it appeared to be of serious potential, we did a much more detailed calculation using a Monte Carlo technique. Then we measured the actual electron intensity in the beam at the second focus and compared the measurement with the calculation.

III. Calculation

Monte Carlo Program

We are interested in the number of secondary electrons generated in the beryllium which reach the second focus of the secondary beam. A Monte Carlo program, originally developed (Nagel, 1965) to simulate electromagnetic cascade showers in lead, was used. The program has been modified at SLAC and elsewhere (Nicoli, 1966) to treat electron energies up to $20 \mathrm{GeV}$. For each incident electron the program calculates the number, type, energy and direction of all secondaries at any point in the absorbing medium. Secondaries are 
generated by bremsstrahlung and pair production and the particles are allowed to scatter and ionize to a low energy cutoff and the absorber thickness desired.

Calculations reported here were done with lead as the target material because of the limitations of the program. We do not believe there would be a significant difference in the results with a beryllium target.

The result of this calculation is a listing (magnetic tape) at each depth selected in the absorber of all the particles present by type, energy and direction. One can then scan the output data at any depth in the absorber and ask for a tabulation by energy of all electrons (positrons, photons) within a certain energy band about each energy point and within a certain angular distribution about $0^{\circ}$ (the forward direction). If one picks the energy band and angular distribution corresponding to the acceptance of the secondary beam transport system, one can obtain the number of secondaries per incident primary that will be accepted at each energy setting of the secondary transport system. It is then assumed that any particle accepted by the secondary system will be transported and thus arrive at the second focus.

IV. Measurement

A. Ion Chamber Description

To measure the electron intensity at the second focus of the muon transport system we constructed a simple parallel plate ionization chamber (see Fig. 3). Because we expected the beam to be rather large $(6 \mathrm{~cm} \times 12 \mathrm{~cm})$ the chamber was necessarily large (approximately $7.5 \times 15 \mathrm{~cm}$ ) with an electrode separation of about $7.5 \mathrm{~cm}$. The chamber was made $3.7 \mathrm{~cm}$ long in the beam direction because we had calibrated a similar, but smaller $(2.5 \mathrm{~cm} \times 2.5 \mathrm{~cm} \times 3.7 \mathrm{~cm})$ 
chamber in an electron beam previously and we wanted a chamber in which the total energy loss would be the same.

To reduce collection losses the chamber was made windowless. However, to obtain stable readings and low background noise levels, due to stray magnetic and $\mathrm{RF}$ fields, the chamber was mounted in a metal box with carbon coated $3 / 4$ mil rubber hydrochloride windows (Fig. 4). The windows on the box were more than 3 inches from the chamber and tests showed there were no collection losses to the windows at this distance. A collecting voltage of $1500 \mathrm{~V}$ was supplied by a battery pack. Chamber current was read on a battery powered Model 602 Keithly electrometer.

\section{B. Ion Chamber Calibration}

To determine the ion chamber response we first calculated the expected response. Since the beam was of very high energy $(6,10,16 \mathrm{GeV})$ and the chamber or absorber was less than $5 \mathrm{~cm}$ of air the energy loss in the chamber is described by the Landau distribution (Landau, 1944; Borsch-Supan, 1961). Figure 5 shows the probability, $f(x, \Delta)$, that an electron will lose an energy $\Delta$ in an $\mathrm{x}=3.7 \mathrm{~cm}$ thick air absorber at $20^{\circ} \mathrm{C}$. The distribution is highly skewed and the most probable energy loss is $7.5 \mathrm{keV}$. 'The chamber lateral dimensions indicate that any delta ray with an energy above $60 \mathrm{keV}$ will most probably escape from the chamber. Hence, the mean energy deposited per electron in the chamber can be defined by

$$
\overline{\epsilon_{L}}=\frac{\int_{0}^{60} \Delta f(x, \Delta) d \Delta}{\int_{0}^{60} f(x \Delta) d \Delta}=9.0 \mathrm{keV}
$$

Alternatively one can calculate the mean energy deposited in the chamber from the restricted stopping power. We calculated the restricted stopping power 
using the Berger and Seltzer formulation (Berger and Seltzer, 1964) and $60 \mathrm{keV}$ as the maximum energy delta ray stopping in the chamber. This leads to a mean energy deposited per electron in the chamber $\left(20^{\circ} \mathrm{C}, 76 \mathrm{~cm} \mathrm{Hg}\right)$ of

$$
\overline{\epsilon_{\mathrm{B}}}=\left(\mathrm{LET}_{\Delta}\right) \rho \mathrm{x}=8.7 \mathrm{keV}
$$

The close agreement between $\overline{\epsilon_{\mathrm{L}}}$ and $\overline{\epsilon_{\mathrm{B}}}$ indicates that either method can be used. For purposes of this paper we will use the Berger \& Seltzer formulation.

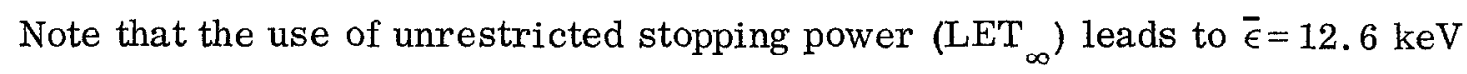
for $10 \mathrm{GeV}$ electrons. This would lead to a $40 \%$ underestimate of the electron fluence in the measurements described later.

Prior to the measurements to be described later, the ion chamber was aligned for calibration in a $16.0 \mathrm{GeV}$ electron beam just ahead of a quantameter which measured the incident electron intensity with a calibration factor of $9.45 \times 10^{13}( \pm 0.3 \%)$ electrons/coulomb (Yount, 1967; Lakin, 1972). The Keithly electrometer was operated in the charge integration mode as was the quantameter readout. For each run the total charge collected on the quantamenter was compared with the total charge collected by the ion chamber. The measurements were repeated several times and an average taken of the results. Measurements were made at three levels of beam intensity $\left(10^{3}, 1.6 \times 10^{4}\right.$ and $4 \times 10^{5}$ electrons/ pulse) to determine the general recombination loss. Data from the $1.6 \times 10^{4}$ electron/pulse point are presented in Table 1. For this data point the average charge collected on the quantameter was $26.55 \times 10^{-9}$ coulombs per $10^{-10}$ coulombs collected by the ion chamber with a fractional standard deviation of the measurements about the mean of $\pm 0.4 \%$. The ion chamber calibration can be written

$$
C_{I C} \text { (electron/coulomb) }=\frac{q_{Q}}{q_{I C}} C_{Q}
$$


where

$$
\begin{aligned}
\mathrm{C}_{\mathrm{Q}}= & \text { quantameter calibration }=9.45 \times 10^{13} \text { electrons } / \text { coulomb } \\
{ }_{\mathrm{Q}} / \mathrm{q}_{\mathrm{IC}}= & \text { ratio of charge integrated on quantameter and ion chamber }, \\
& \text { respectively. }
\end{aligned}
$$

Data for the three levels of beam intensity are shown in Table 2. (We have assumed that there are no recombination effects in the quantameter (Yount, 1973).)

The ideal response (100\% collection efficiency) can be calculated according to the equation

$$
\begin{aligned}
(\mathrm{n} / \mathrm{q})_{\text {ideal }} & =\frac{\mathrm{W}}{1.6 \times 10^{-19} \bar{\epsilon}_{\mathrm{B}}} \\
& =\frac{33.7}{1.6 \times 10^{-19}\left(8.7 \times 10^{3}\right)} \\
& =2.42 \times 10^{16} \text { electrons/coulomb } .
\end{aligned}
$$

Collection efficiency at each beam intensity can then be calculated from the measured calibration (Table 2) and the ideal response, the results are shown in Fig. 6. Curve A in Fig. 6 was calculated from the theory of recombination loss in a pulsed beam (Boag, 1966) and normalized to $93.5 \%$ (intrinsic) efficiency which is the average of the data at $10^{3}$ and $1.6 \times 10^{4}$ electrons/pulse where recombination losses were not in evidence (within the statistics).

Boag's theory states that in a pulsed radiation field where the pulse is short and the interval between pulses long compared with the transit time of ions across the chamber, the collection efficiency can be calculated from:

$$
f=\frac{1}{u} \ln (1+u)
$$


where

$$
\begin{aligned}
\mathrm{u}= & \mu \frac{\mathrm{rd}}{\mathrm{V}} \\
\mathrm{r}= & \text { initial charge density liberated per pulse }\left(\mathrm{esu} / \mathrm{cm}^{3}\right) \\
\mathrm{d}= & \text { collecting plate separation }(\mathrm{cm}) \\
\mathrm{V}= & \text { collecting voltage (volts) } \\
\mu= & \text { constant related to ion mobility and given as } 1090 \mathrm{~V} \mathrm{~cm} / \mathrm{esu} \\
& \text { for air at STP (Boag, 1966). }
\end{aligned}
$$

The $\mathrm{d}^{2}$ term arises from two sources. One is the field gradient V/d and the other is the initial charge overlap distance. Both enter explicitly in the equation that describes the overlap region as it contracts with time. In a radiation field larger than the chamber, the initial region of charge overlap is equal to the collector separation distance, $d$, and Boag's formulation adequately describes the situation. However, when the radiation field is very much smaller than the ion chamber, as in our case, the theory must be modified. We find that

$$
\mu=\mu \frac{\mathrm{rDd}}{\mathrm{V}}
$$

where

$$
\mathrm{D}=\text { initial overlap distance }(\mathrm{cm}) \text {. }
$$

For our calibration conditions, D was determined from photographs of the beam and was found to be $0.15 \mathrm{~cm}$; whereas d was measured to be $7.63 \mathrm{~cm}$. Similar calculations for different beam sizes were made for correcting the beam measurements discussed below. These are also shown in Fig. 6 (curves $\mathrm{B}$ and $\mathrm{C}$ ).

The beam used in the calibrations was necessarily very small because of the small diameter beam pipe between the ion chamber and the quantameter. 
However, the beam used in the later measurements was considerably larger. Since it was conceivable that collection losses (e.g., losses to the chamber walls) might be greater for larger beam sizes, a test was made to determine whether the intrinsic collection efficiency of $93.5 \%$ (defined here as a measure of losses other than by recombination)changed as a function of beam size . This check was done both in the electron beam and with a collimated beam of ${ }^{137} \mathrm{Cs}$ gamma rays. Beam sizes ranging from $0.10 \mathrm{~cm} \times 0.15 \mathrm{~cm}$ to $5 \mathrm{~cm} \times 10 \mathrm{~cm}$ were checked and no change in collection efficiency was noted. C. Beam Parameters and Profile

A primary electron beam was established at $19.8 \mathrm{GeV}, 5$ pulses per second, $0.5 \times 10^{10}$ electron/pulse incident on the $\pi$ production target shown in Fig. 1 . The beam was then adjusted to pass through the $\pi$ target and a secondary emission monitor (SEM) centered on the face of the 8.25 radiation length thick Be filter. This aligned the beam directly into the muon beam channel. The beam was then moved horizontally just enough to miss the $\pi$ target.

Magnets in the muon beamline were set to transport $6 \mathrm{GeV}$ particles for the first measurement, $10 \mathrm{GeV}$ for the second, and $16 \mathrm{GeV}$ for the third. A typical $\mathrm{X}$-ray film photograph of the beam profile at the second focus is shown in Fig. 7. The outline of the ion chamber is also shown to indicate the position of the beam in the chamber. Beam and chamber alignment was initially done using Polaroid film that was positioned at a known location relative to the ion chamber and then exposed. These pictures allowed us to adjust the chamber position and/or the secondary beam steering to bring the beam cleanly through the chamber, as shown in Fig. 7. 


\section{Results}

The purpose of the measurements was to determine whether Monte Carlo calculations accurately predict the electron beam intensity in a secondary beamline set to transport particles at various energies. Consequently the measurements were made at three separate secondary transport energies $(6,10$ and $16 \mathrm{GeV})$. At each energy point the secondary beam steering was adjusted as described above and an X-ray film was exposed (downstream from the chamber) while the average ion chamber current was read for the data point. Figure 7 is one such exposure. Because the secondary electron intensity decreases as the transported energy increases, the primary beam intensity had to be increased to $5.0 \times 10^{10}$ electrons/pulse at the $16 \mathrm{GeV}$ data point. The results of the measurements are given in Table 3. Accuracy of the current reading was $\pm 5 \%$. Background current on the ion chamber was $5 \times 10^{-14} \mathrm{amp}$.

During these measurements the air temperature was close to $0^{\circ} \mathrm{C}$ and the mean energy deposited per electron in the chamber was calculated, as discussed above, to be $\bar{\epsilon}=9.3 \mathrm{keV}$. This value of $\bar{\epsilon}$ was used to compute the electron intensity from the ion chamber current as follows: The first step is to determine if there are any recombination losses to be considered. This was done by calculating the number of electrons per pulse in the ion chamber assuming no recombination loss using equation (4) with $\bar{\epsilon}=9.3 \mathrm{keV}$ and Table 3. By estimating the size of the beam from the photographs, the expected collection efficiency was then determined from Fig. 6 and the number of electrons per pulse was re-calculated. Two or three iterations were sufficient to correct for losses in all cases. From the photographs of the beam curve $\mathrm{B}$ was chosen for the 6 and $16 \mathrm{GeV}$ measurement while curve $\mathrm{C}$ was 
chosen for the $10 \mathrm{GeV}$ measurement. Results are shown in Table 4. We estimate that the measurements are accurate to $\pm 10 \%$ with the estimated uncertainties breaking down as shown in Table 5 .

The secondary beamline aperture was rectangular such that it provided an angular acceptance of 2 milliradians horizontally and 10 milliradians vertically about $0^{\circ}$ with respect to the incident beam. Secondary transport momentum acceptance was determined by fitting the measured beam spot using a beam transport code called LURCH (Stork, 1972). This was found to be $\pm 6 \%$ about the set energy point. With this information the results of the Monte Carlo calculation were scanned to select all electrons emerging from an 8 radiation length thick absorber within $\pm 6 \%$ about a specified energy and within the given angular constraints. Since the absorber used in the measurements was 8.25 radiation lengths, the Monte Carlo data calculated for 8.00 radiation lengths were corrected by $\exp -\frac{7}{9 \mathrm{X}_{0}}\left(0.25 \mathrm{X}_{0}\right)$ where $\mathrm{X}_{0}$ is the number of radiation lengths in the absorber and $7 / 9 \mathrm{X}_{0}$ is the asymptotic pair production cross section. This assumes that all the electrons emerging from the absorber arise from pair production of high energy photons in the last portion of the absorber. Thus the number of electrons emerging will be reduced as the high energy photons are attenuated. For small corrections, as in the above situation, Monte Carlo studies have demonstrated that the model works.

The ratio of the secondary to primary electron number, $\mathrm{N}_{\mathrm{S}} / \mathrm{N}_{0}$, is plotted in Fig. 8 as a function of the secondary energy, $E_{S}$, where the three experimentally determined points are compared with the Monte Carlo predictions. Error bars associated with the measurements are based on the estimated uncertainty $( \pm 10 \%)$ while error bars on the Monte Carlo points were obtained by calculating the standard deviation about the mean of a number of Monte Carlo calculations at each energy. 


\section{Discussion}

It is obvious from Fig. 8 that there is significant disagreement between the calculation and the measurement at the lower energies. We have not been able to refine the calculation in any logical way to remove this discrepancy. One possible explanation is that the primary electron beam was slightly missteered so that the secondary beam channel was accepting particles at some angle greater than zero degrees to the incident beam. Introducing a one milliradian horizontal offset in the calculation reduced the number of 1 to $5 \mathrm{GeV}$ electrons by about $15 \%$. We do not believe the missteering could have been greater than one milliradian; so this will not account for the entire discrepancy. Another possible explanation is that the secondary beam transport magnets were slightly misadjusted for the energy being transported and some of the beam was lost in the secondary system. There is no way to check this possibility by adjusting the calculation.

In any case, the Monte Carlo calculation provides an upper limit to the particle flux density to be expected in a secondary beam. The data generated by these calculations can be reliably used in designing the safety of high energy secondary beam transport lines. 


\section{$\underline{\text { References }}$}

Berger, M. J. and Seltzer, S. M., 1964, "Tables of Energy Losses and Ranges of Electrons and Positrons" (NASA SP-3012), National Aeronautics and Space Administration, Washington, D. C.

Boag, J. W., 1966, Radiation Dosimetry, Vol. II: Instrumentation, F. H. Attix and W. C. Roesch, eds. (New York: Academic Press), Chapter 9: "Ionization Chambers."

Borsch-Supan, W., 1961, J. Res. Nat. Bur. Standards - B. Math., Math. Phys. 65B, 245.

Lakin, W., 1972, personal communication.

Landau, L., 1944, J. Phys. 8, 201.

Nagel, H. H., 1965, Z. fur Physik 186, 319; and Stanford Linear Accelerator Center translation No. SLAC-TRANS-28.

Nicoli, D. F., 1966, "The Application of Monte Carlo Cascade Shower Generation in Lead in the Several BeV Energy Region, "Massachusetts Institute of Technology, unpublished.

Stork, D., 1972, University of California, Los Angeles report, unpublished. Yount, D., 1967, Nucl. Instr. Meth. 52, 1. Yount, D., 1973, personal communication. 
Table 1

Integrated Charge

\begin{tabular}{cc}
\hline $\begin{array}{c}\text { Quantameter } \\
\left(10^{-9} \text { coulombs }\right)\end{array}$ & $\begin{array}{c}\text { Ion Chamber } \\
\left(10^{-10} \text { coulombs }\right)\end{array}$ \\
\hline 28.08 & 1.05 \\
28.18 & 1.05 \\
28.00 & 1.05 \\
139.86 & 5.31 \\
55.68 & 2.13 \\
\hline
\end{tabular}

Table 2

Ion Chamber Calibration Data

\begin{tabular}{lccc}
\hline $\begin{array}{c}\text { Beam Intensity } \\
\text { (electrons/pulse) }\end{array}$ & $\mathrm{q}_{\mathrm{Q}} / \mathrm{q}_{\mathrm{IC}}$ & $\begin{array}{c}\text { Fractional } \\
\text { Standard } \\
\text { Deviation }\end{array}$ & $\left.\begin{array}{c}\text { Ion Chamber Calibration } \\
\text { /electrons } \\
(\text { coulomb }\end{array}\right)$ \\
$(1.0 \pm 0.5) \times 10^{3}$ & 281.8 & $\pm 0.06 \%$ & $2.66 \times 10^{16}$ \\
$(1.6 \pm 0.4) \times 10^{4}$ & 265.5 & $\pm 0.4 \%$ & $2.51 \times 10^{16}$ \\
$(4 \pm 1) \times 10^{5}$ & 393.4 & $\pm 1.7 \%$ & $3.72 \times 10^{16}$ \\
\hline
\end{tabular}


Table 3

Secondary Electron Beam Intensity

\begin{tabular}{ccc}
\hline $\begin{array}{c}\text { Primary Beam Current } \\
\text { (electrons/second) }\end{array}$ & $\begin{array}{c}\text { Secondary Beam } \\
\text { Energy } \\
(\mathrm{GeV})\end{array}$ & $\begin{array}{c}\text { Ion Chamber Current } \\
\text { (amp) }\end{array}$ \\
$2.5 \times 10^{10}$ & 6 & $1.0 \times 10^{-10}$ \\
$2.5 \times 10^{10}$ & 10 & $4.8 \times 10^{-11}$ \\
$2.5 \times 10^{11}$ & 16 & $3.9 \times 10^{-11}$ \\
\hline
\end{tabular}

Table 4

Number of Secondary Electrons per Incident Primary

\begin{tabular}{ccc}
\hline $\begin{array}{c}\text { Secondary Flectron Energy } \\
(\mathrm{GeV})\end{array}$ & $\begin{array}{c}\text { Secondary Electrons } \\
\text { per Pulse }\end{array}$ & $\begin{array}{c}\text { Ratio of Secondary to } \\
\text { Primary Electrons, } \mathrm{N}_{\mathrm{S}} / \mathrm{N}_{0}\end{array}$ \\
\hline 6 & $5.1 \times 10^{5}$ & $(1.0 \pm 0.1) \times 10^{-4}$ \\
10 & $2.4 \times 10^{5}$ & $(4.8 \pm 0.5) \times 10^{-5}$ \\
16 & $1.9 \times 10^{5}$ & $(3.8 \pm 0.4) \times 10^{-6}$ \\
\hline
\end{tabular}

Table 5

Estimated Uncertainties

\begin{tabular}{ll}
\hline Ion chamber calibration & $\pm 7 \%$ \\
Energy loss calculation & $\pm 3 \%$ \\
Beam measurement & $\pm 5 \%$ \\
Recombination correction & $\pm 5 \%$ \\
\hline
\end{tabular}




\section{Figure Captions}

1. Vertical ray trace of primary electron beams.

2. Vertical ray trace of secondary muon (electron) beam.

3. Photograph of parallel plate ionization chamber.

4. Photograph of ionization chamber inside metal box (without rubber hydrochloride window).

5. Landau distribution of energy loss in $3.7 \mathrm{~cm}$ of air at $20^{\circ} \mathrm{C}$.

6. Ion chamber collection efficiency as a function of the beam intensity (electrons/pulse) for various beam dimensions.

7. Photograph of $10 \mathrm{GeV}$ secondary beam relative to the chamber position.

8. Ratio of secondary to primary electron number as a function of secondary energy. 


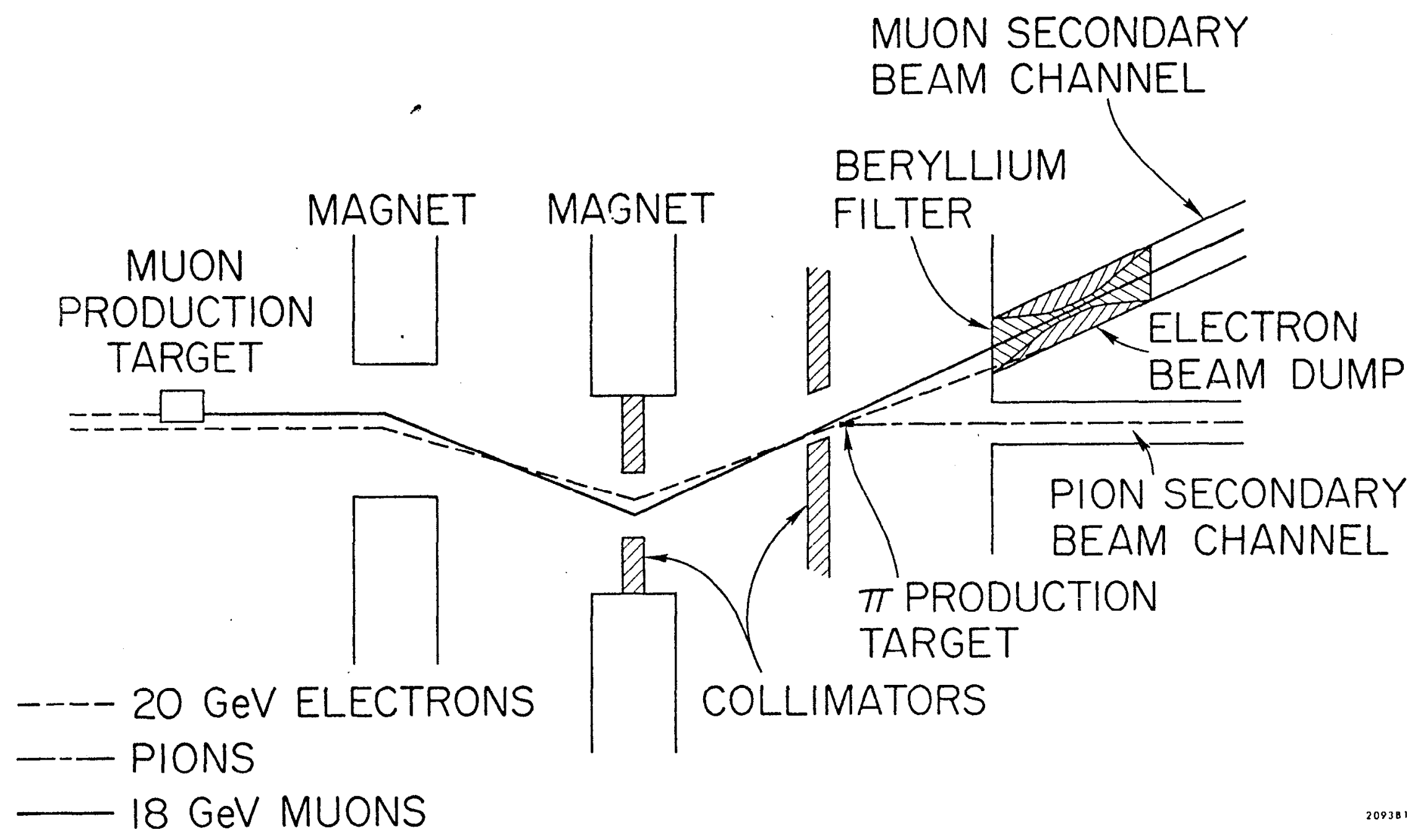

Fig. 1 




Fig. 2 


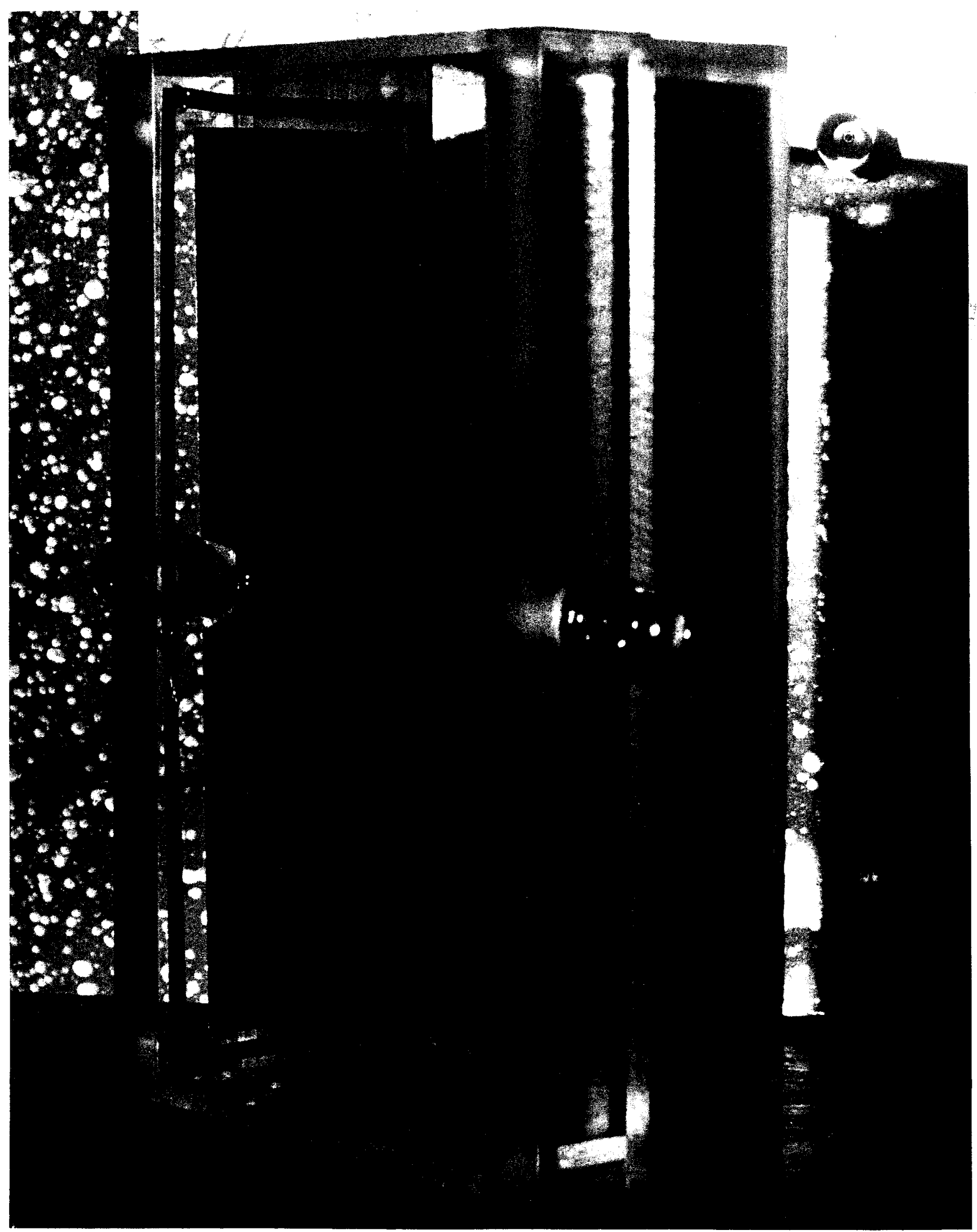

Fig. 3 


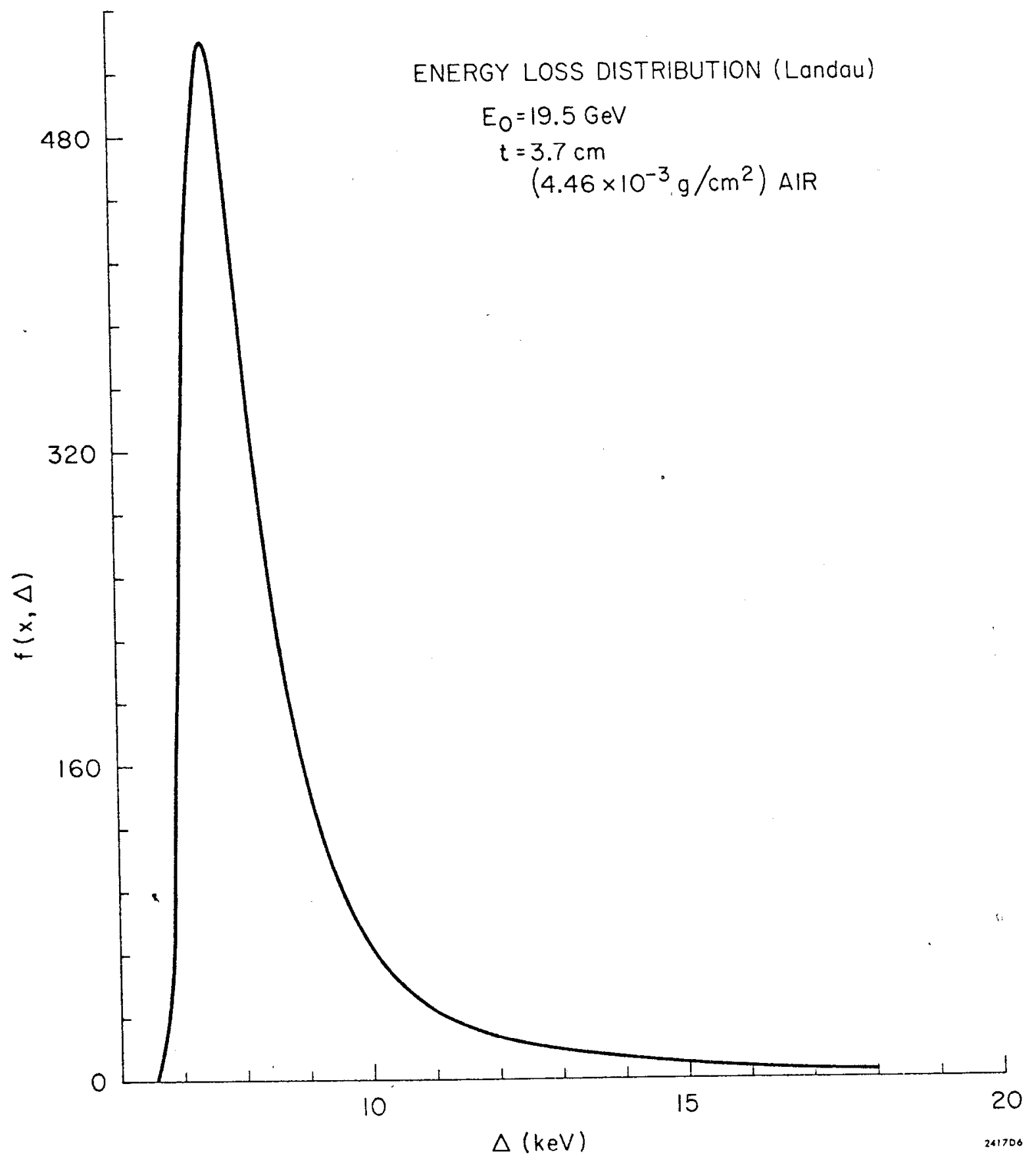

Fig. 5 




, Fig. 6 
$10 \mathrm{GeV}$



Fig. 7 


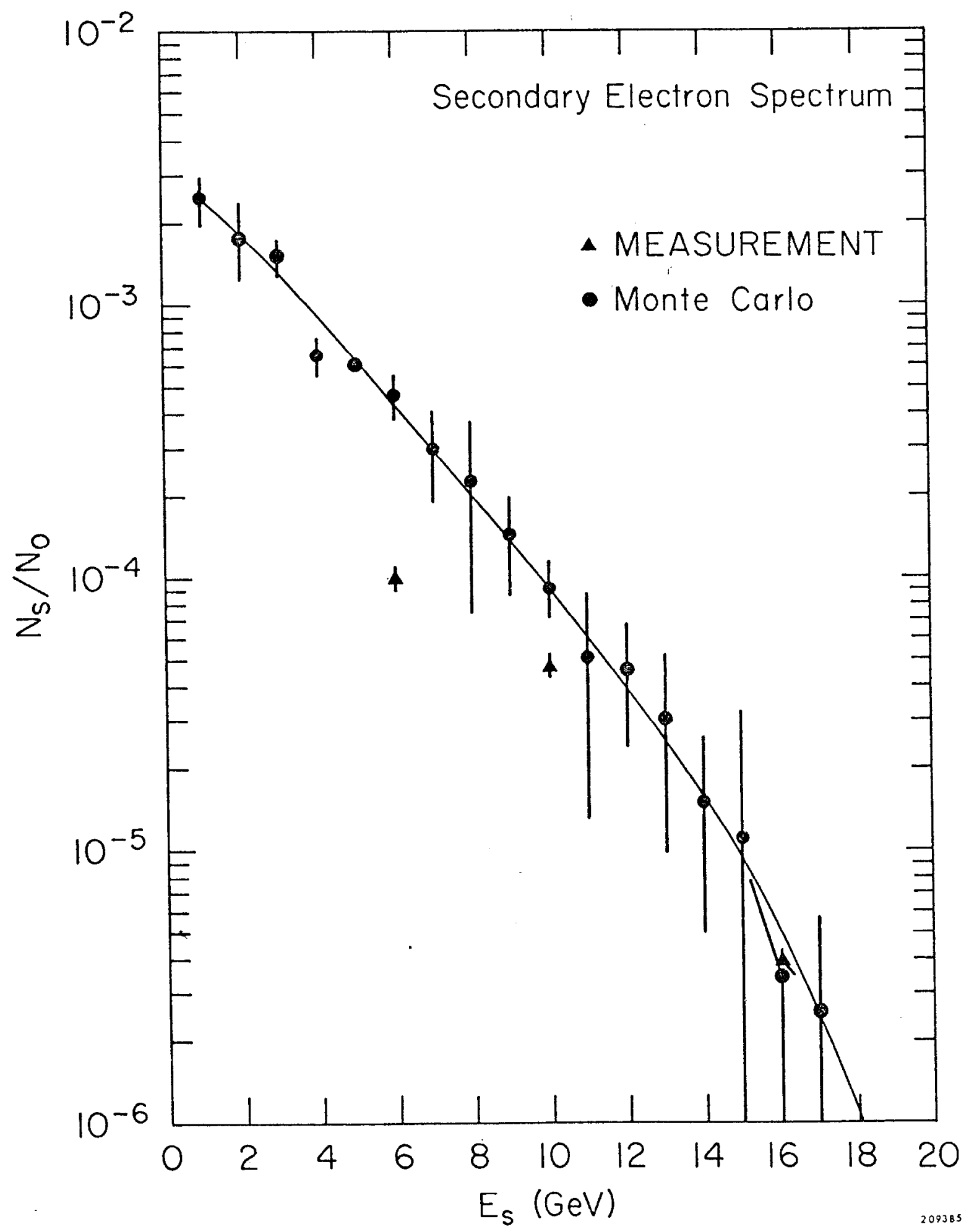

Fig. 8 